\title{
Influence of External Currents in Sensors Based on PCB Rogowski Coils
}

\author{
Artero J.A. ${ }^{1}$, Arcega F.J. ${ }^{2}$, \\ ${ }^{1}$ E.R.Z. Endesa Distribución \\ C/ San Miguel 1, 50001 Zaragoza \\ ${ }^{2}$ Department of Electrical Engineering \\ Escuela Universitaria de Ingeniería Técnica Industrial de Zaragoza (E U I T I Z.), University of Zaragoza \\ Rio Ebro Campus - María de Luna, nº 3 Ed. “Torres Quevedo”, 50018 Zaragoza (Spain) \\ phone:+34 976 762169, fax:+34 976 762226, e-mail: arcegafj@unizar.es
}

\begin{abstract}
A current sensor based in the Rogowski coil is an innovative measuring system that gives advantages with respect to conventional measuring systems based in current transformers with magnetic core [1], [2].and [3]. Their main advantages are: the linearity, the span and the bandwidth.

Different kinds of manufacturing allow to obtain an important variety of Rogowski coils with different properties. One way of manufacturing is using the same method as for producing printed circuit boards, so by this way is possible to produce coils very similar and with a high precision.

The authors are working in current measurement with Rogowski coils or Hall effect sensors and in particular in the realization of good and accurate coils [4] and [5]. In this work, the influence of external currents to the coil in the measured current by the coil has been evaluated.
\end{abstract}

Key words: Rogowski coils, current measurement, sensors

\section{Introduction}

One interesting alternative to the classical Rogowski coils manufactured with conductor cable is to manufacture the coil like a printed circuit board (PCB) [6], [7] and [8]. The main advantages of this coil are :

a) Easy to manufacture

b) Very cheap y light

c) Good linearity and bandwidth

The main inconvenience is that the step of each turn is relatively high with respect to a conventional coil, because the number of turns in a PCB coil is lower than in a conventional coil. This is the weakest point of this kind of coils because the errors produced by external currents (or external varying magnetic fields) in the vicinity of the coil can more easily introduce an error in the measurement.

We have tested different configurations in the shape of the turns of the coil for minimizing the error. An acceptable result has been obtained placing two coils in parallel position and very closer together, whose turns are in opposite direction. With an electrical connection in the appropriate way, the external magnetic fields cancel in the system of the two coils.

More closer the coils are and more symmetrical his position, better is the measurement and lower the error produced by external currents or magnetic fields.

\section{Rogowski coil construction}

There are two main ways to manufacture a Rogowski coil. The classical one consisting in wrapping in a flexible core (non magnetic) the cable of the coil. The second one more innovative, consists in "wrapping" in a hard core the turns of the cable. In that case is better to use the techniques of the printed circuit board manufacturing, using the two sides of the board. Each side of the board has part of the circuit of the turn. The junction of the two sides is through the board and by means of a welding. Industrial techniques are better than research ones and in the production process smaller coils can be produced than in the research process.

The final result is an helicoidal coil, shown in figure 1, similar to a conventional Rogowski coil but the width is determined by the width of the PCB and a very well known geometry that is very easy to simulate in order to study the electromagnetic problem.

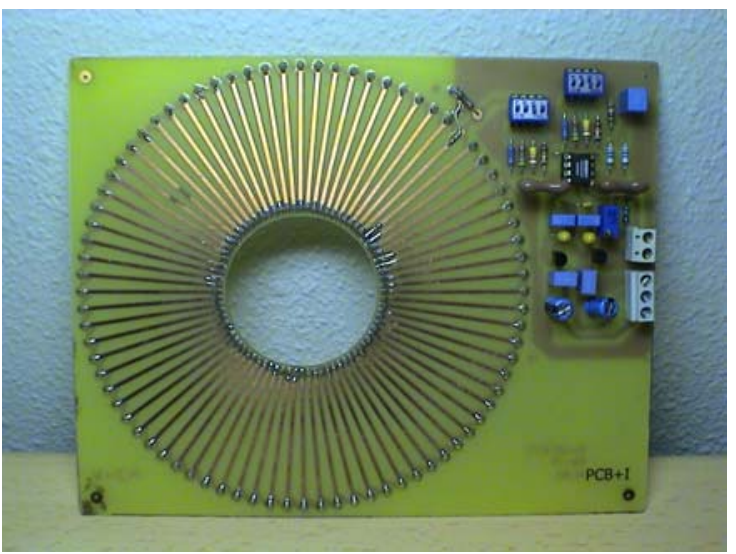

Fig. 1. PCB Rogowski coil plus integrator circuit 
The working principle of this PCB coils is the same as for the flexible coils and has been explained in the literature [1]. The current to be measured (varying with the time) gives rise to a magnetic flux changing with the time that is translated to a voltage in the terminals of the Rogowski coil, proportional to the rate of current change

$\left(\frac{d i}{d t}\right)$.

The main characteristics of this kind of coils in comparison with conventional ones are:

- A great accuracy. The way to manufacture the coil allow to have a coil with dimensions and shape of the turns very constant in all the coil.

- A lower value of auto-inductance L for the same value of the mutual inductance $\mathrm{M}$ in comparison with a flexible coil. So the response of the coil and the bandwidth are improved.

- Easy manufacturing and low cost. These coils are very interesting for several applications as for instance in protection of power electric systems where they are substituting the conventional current transformers with magnetic core

\section{Errors in the measurement with PCB Rogowski coils}

The helix of the turn for conforming the Rogowski coil defines a section in the space. The magnetic flux that crosses this section produces the electromotive force (voltage) proportional to the rate of change of the current to be measured and that crosses through the coil.

The problem is that this section is not a flat area and external currents in the vicinity of the sensor can cross by part of the circuit and not be entirely cancelled by other part of the circuit so deriving in an error of the measurement. All that voltages produced by the presence of sections of the surface of the turn affected by external currents are a source of error in the current measurement.

In general we can deduce that lower is the step of advance of the turn in the coil, flatter is the section of surface of the turn and lower is the effect of in the measurement (lower error).

Details of several turns of a Rogowski coil and the decomposition of the surface into three surfaces in the three axis is shown in the figure 2 . The conductor of the turn in the upper side of the PCB with the conductor in the lower side define a surface non flat. Such surface can be projected in the three different directions of a coordinate system, giving as consequence, the sections defined in the figure 2 as section I, II and III and that will be employed for the simulation realized for obtaining part of the results of this work.

The values shown in the figure 2 are

- $\mathrm{R}_{\mathrm{e}}$ is the external radius of the coil

- $\mathrm{R}_{\mathrm{i}}$ is the internal radius

- $\mathrm{s}$ is the width of the board.

In the case of having a conductor carrying a current to be measured, centred in the coil, perpendicular to the plane determined by the coil, the flux of the magnetic field originated by the current will be created through the surfaces indicated by sections II and III in the figure 2.

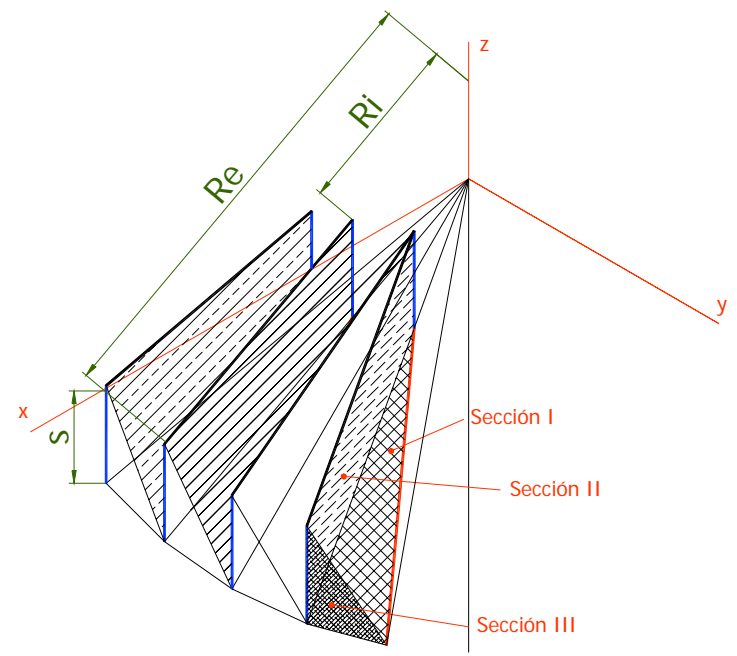

Fig. 2. Detail of a PCB Rogowski coil.

We have built and simulated a series of prototypes of PCB Rogowski coils with 100 turns. The width is $1.6 \mathrm{~mm}$, the external radius is $87.4 \mathrm{~mm}$ and the internal radius is $40 \mathrm{~mm}$.

The test have been carried out in our laboratory. We have a strait conductor of $2 \mathrm{~m}$ length capable of carrying a current between 5 and $1000 \mathrm{~A}$ and with a frequency of $50 \mathrm{~Hz}$. The conductor has an external diameter of $19 \mathrm{~mm}$ being hollow in the inner. For obtaining the current range we use a transformer whose primary is connected to the electrical network. The secondary is short-circuited through the main straight conductor and bus bars.

For simulation a mathematical software has been used. After the simulation of the effect of external currents to the coil, we can conclude that

- A conductor situated in the plane determined by the coil and in the vicinity, originates a voltage similar to the voltage produced by the same current in the inner of the coil. The magnetic flux through the different sections is shown in the figure 3

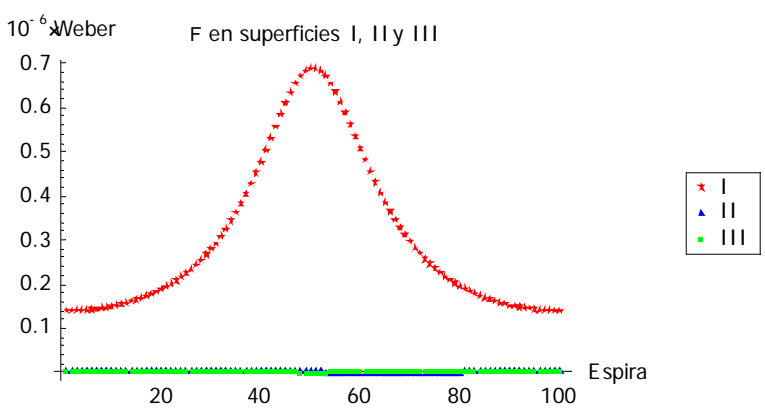

Fig. 3. Magnetic flux for each section of the turn produced by a cable carrying a current in the plane of the coil

- A conductor situated perpendicularly to the plane of the coil and external to it but in its vicinity originates a lower error than in the previous case because the section crossed by the unwanted magnetic field, defined as section III is much lower. 
For the case of a conductor situated in the interior of the coil but not perpendicular or not centred gives rise to a magnetic flux non uniform or constant in the three sections of the turns in function of the angle. Globally the induced voltage does not differ from the one produced by the cable centred and perpendicular because there is an effect of compensation between the different turns that compose the coil.

After the simulation for obtaining the flux for each section and for different configurations of the conductor currying the current we obtain that, if the conductor is situated in the plane of the coil and in the neighbourhood, the induced voltage (for the same current $I_{0}$ ) is similar to the one created by an internal conductor currying the same current.

For the case of a conductor contained in the plane of the coil and situated to a distance of his centre, the flux has been shown in figure 3.

In the case of an internal conductor, perpendicular to the coil, the magnetic flux in the sections II of each coil is constant and the flux in the sections I and III is practically zero as is shown in figure 4.

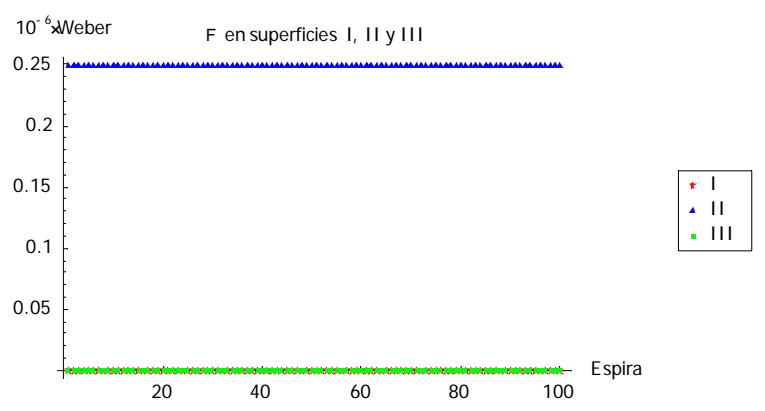

Fig. 4. Magnetic flux for each section of the turn produced by a cable carrying a current perpendicular to the plane of the coil

In the case of a conductor situated outside the coil and in the plane defined by the Rogowski coil, the magnetic flux through the sections changes with the different turns depending on its position with respect to the conductor (closer or farther). The global result for all the coil is that the induced voltage is similar to the former case with the internal conductor and being origin to the inadmissible errors.

The experimental results show that the influence of external conductor in the voltage induced in the coil decreases with the distance as shown in figure 5

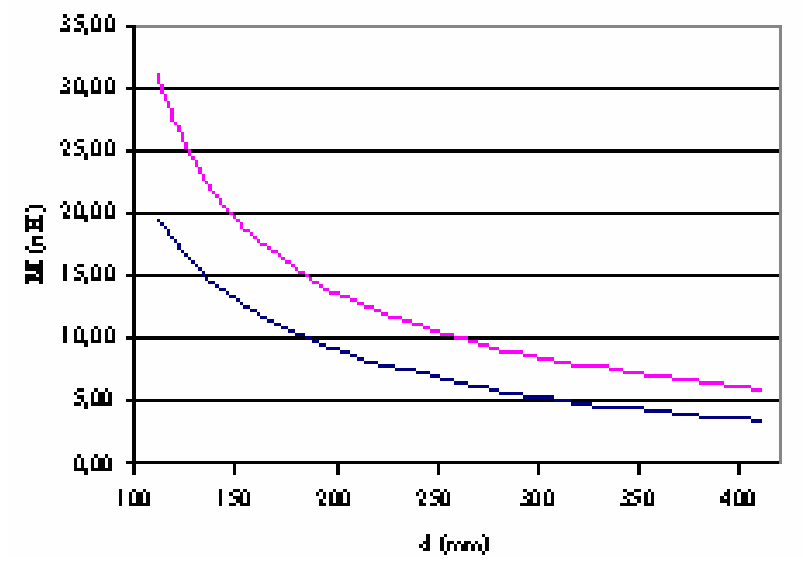

Fig. 5. Experimental results for inductance versus distance of the external conductor with respect to the coil

\section{Conclusions}

From the experience obtained for different prototypes of Rogowski coils constructed in flexible way or in PCB we can conclude that

- Coils constructed in PCB are more sensible with respect to external magnetic fields. This is a consequence of the big ratio between the external and internal radius of the turns that conform the coil for maintaining the same characteristics of the flexible coil.

- The number of turns per meter that can be incorporated in a PCB is much smaller than in the case of a flexible coil. Moreover a lower number of turns per meter gives origin to a bigger step between turns, in consequence the section I, defined in previous paragraph, is more important. This section is the origin of errors due to his size is comparable to section II (in the PCB), so the effect of external currents is similar to the internal ones for the same value of current

- For the case of flexible coils the helicoidal advance of the turns is much lower (because of the bigger number of turns) so the section I is smaller and in consequence the errors produced by external currents are smaller. No so smaller as could be expected because of the big number of turns increases the amount of the error but always is lower than the one produced in the PCB.

One way of avoiding the inconvenient mentioned for the PCB coils will be the construction of the turns in the PCB so smaller as technically possible for increasing the number of turns per meter and reducing the ratio between the external and internal radius.

For reducing the surface of the section I another way is to change the shape of the conductor in the PCB as shown in the figure 5. In such way is possible to minimise the section I because the go and back of the current in the surface of the PCB is the same in almost all the turn

In the constructed and tested prototypes this small modification in the conductors in the PCB has produced an important reduction in the influence of the external current carrying conductor.

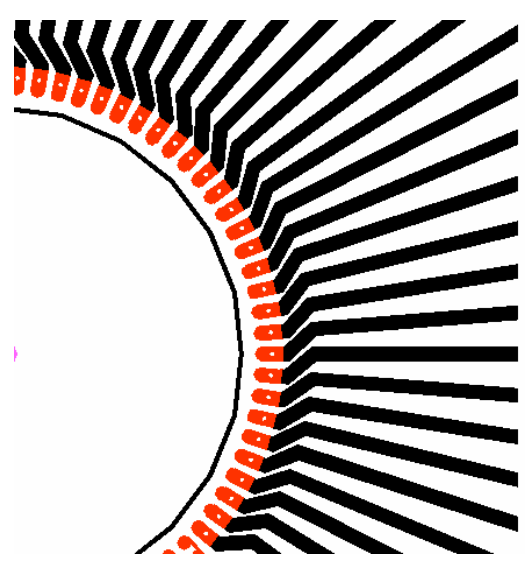

Fig. 5. New model for construction of a PCB Rogowski coil . 
Another way of minimising the influence of external magnetic fields is superpose two PCB coils with the turns in opposite way, as shown in figure 6 . In this way the magnetic fluxes crossing the section I in each coil have opposite sign for the external conductors giving a result near zero. For internal conductors the magnetic fluxes through the section II have the same sign and the induced voltage in the coil is the double

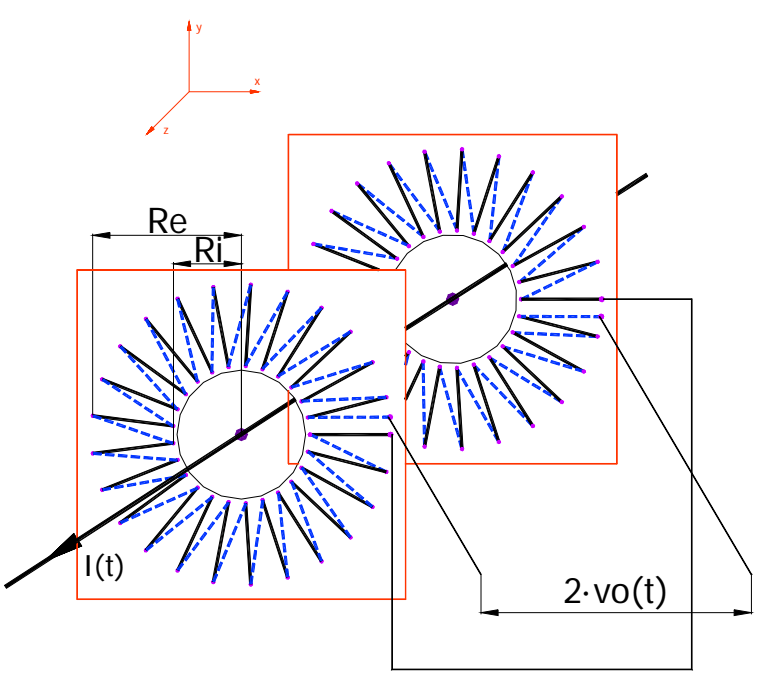

Fig. 6. New model with two coils in opposite way for reducing the influence of external conductors

Now we are working in the experimental testing of the prototypes indicated for evaluating more in detail the effect of external conductors in the vicinity of the current sensors based on the Rogowski coil.

\section{References}

1. W. F. Ray \& C.R. Hewson. "High performance Rogowski current transducers”. Power Electronic Measurements Ltd. 2000.

2. Nicolas Karrer \& Patrick Hofer-Noser. “A new current measuring principle for power electronic applications”. Electrical Engineering and design lab (EEK), Swiss Federal Institute of Technology, Zurich. 1999.

3. R. Gross, H.J. Herrmann, U. Katschinski, P. Menke, A. Ostermeier, J. Schmid, M. Wache. "Substation control and protection system for novel sensors". Cigré 12/23/34-03 2000.

4. F. J. Arcega and J. A. Artero "Current sensor based on Rogowski coils” in Proc ICREPQ Barcelona 2004

5. J. Letosa, J. S. Artal, M. Samplon, A. Uson and F. J. Arcega "Modelisation of Current sensors by finite element methods" Measurement Vol 35, pp. 233-241 (2004)

6. Ljubomir Kojovic. "PCB Rogowski Coils Benefit Relay Protection”. IEEE Computer Applications in Power, July 2002.

7. Ljubomir Kojovic. "High-Precision Rogowski Coils for Improved relay protection, control and measurements”. Cooper Industries, Inc. Bulletin number 02049. 2002.

8. John D. Ramboz. "Machinable Rogowski coil, design and Calibration". IEEE Transactions on Instrumentation and measurement $\mathrm{Vol} 45, \mathrm{~N}^{\circ} 2$, April 1996. 